Proyecciones Journal of Mathematics

Vol. 31, No 3, pp. 209-217, September 2012.

Universidad Católica del Norte

Antofagasta - Chile

\title{
Bounded linear operators for some new matrix transformations
}

\author{
M. $A I Y U B$ \\ University of Bahrain, Kingdom of Bahrain \\ Received: March 2012. Accepted : April 2012
}

\begin{abstract}
In this paper, we define $(\sigma, \theta)$-convergence and characterize $(\sigma, \theta)$ conservative, $(\sigma, \theta)$-regular, $(\sigma, \theta)$-coercive matrices and we also determine the associated bounded linear operators for these matrix classes.
\end{abstract}

AMS Subject Classification (2000) : 46A45, $40 H 05$.

Keywords and phrases : Sequence spaces; invariant mean; matrix transformation; bounded linear operators. 


\section{Introduction and preliminaries}

We shall write $w$ for the set of all complex sequences $x=\left(x_{k}\right)_{k=0}^{\infty}$. Let $\phi, \ell_{\infty}, c$ and $c_{0}$ denote the sets of all finite, bounded, convergent and null sequences respectively; and $c s$ be the set of all convergent series. We write $\ell_{p}:=\left\{x \in w: \sum_{k=0}^{\infty}\left|x_{k}\right|^{p}<\infty\right\}$ for $1 \leq p<\infty$. By $e$ and $e^{(n)}(n \in \mathbf{N})$, we denote the sequences such that $e_{k}=1$ for $k=0,1, \ldots$, and $e_{n}^{(n)}=1$ and $e_{k}^{(n)}=0(k \neq n)$. For any sequence $x=\left(x_{k}\right)_{k=0}^{\infty}$, let $x^{[n]}=\sum_{k=0}^{n} x_{k} e^{(k)}$ be its $n$-section.

Note that $c_{0}, c$, and $\ell_{\infty}$ are Banach spaces with the sup-norm $\|x\|_{\infty}=\sup _{k}\left|x_{k}\right|$, and $\ell_{p}(1 \leq p<\infty)$ are Banach spaces with the norm $\|x\|_{p}=\left(\sum\left|x_{k}\right|^{p}\right)^{1 / p}$; while $\phi$ is not a Banach space with respect to any norm.

A sequence $\left(b^{(n)}\right)_{n=0}^{\infty}$ in a linear metric space $X$ is called Schauder basis if for every $x \in X$, there is a unique sequence $\left(\beta_{n}\right)_{n=0}^{\infty}$ of scalars such that $x=\sum_{n=0}^{\infty} \beta_{n} b^{(n)}$.

Let $X$ and $Y$ be two sequence spaces and $A=\left(a_{n k}\right)_{n ; k=1}^{\infty}$ be an infinite matrix of real or complex numbers. We write $A x=\left(A_{n}(x)\right), A_{n}(x)=\sum_{k}$ $a_{n k} x_{k}$ provided that the series on the right converges for each $n$. If $x=\left(x_{k}\right)$ $\in X$ implies that $A x \in Y$, then we say that $A$ defines a matrix transformation from $X$ into $Y$ and by $(X, Y)$ we denote the class of such matrices.

Let $\sigma$ be a one-to-one mapping from the set $\mathbf{N}$ of natural numbers into itself. A continuous linear functional $\varphi$ on the space $\ell_{\infty}$ is said to be an invariant mean or a $\sigma$-mean if and only if (i) $\varphi(x) \geq 0$ if $x \geq 0$ (i.e. $x_{k} \geq 0$ for all $k$ ), (ii) $\varphi(e)=1$, where $e=(1,1,1, \cdots)$, (iii) $\varphi(x)=\varphi\left(\left(x_{\sigma(k)}\right)\right)$ for all $x \in \ell_{\infty}$.

Throughout this paper we consider the mapping $\sigma$ which has no finite orbits, that is, $\sigma^{p}(k) \neq k$ for all integer $k \geq 0$ and $p \geq 1$, where $\sigma^{p}(k)$ denotes the $p$ th iterate of $\sigma$ at $k$. Note that, a $\sigma$-mean extends the limit functional on the space $c$ in the sense that $\varphi(x)=\lim x$ for all $x \in c$, (cf [10]). Consequently, $c \subset V_{\sigma}$, the set of bounded sequences all of whose $\sigma$-means are equal.We say that a sequence $x=\left(x_{k}\right)$ is $\sigma$-convergent if and only if $x \in V_{\sigma}$.

$$
V_{\sigma}=\left\{x \in \ell_{\infty}: \lim _{p \rightarrow \infty} t_{p n}(x)=L, \text { uniformly in } \mathrm{n}\right\} .
$$

where $L=\sigma-\lim x$, where

$$
\mathrm{t}_{p n}(x)=\frac{1}{p+1} \sum_{m=0}^{p} x_{\sigma^{m}(n)},
$$

Using the concept of Schaefer [17] defined and characterized the $\sigma$ conservative, $\sigma$ - regular and $\sigma$-coercive matrices. If $\sigma$ is translation then 
the $\sigma$ - mean often called Banach Limit [2] and the set $V_{\sigma}$ reduces to the set $f$ of almost convergent sequence studied by Lorenz [9]. By a lacunary sequence we mean an increasing sequence $\theta=\left(k_{r}\right)$ of integers such that $k_{0}=0$ and $h_{r}=k_{r}-k_{r-1} \rightarrow \infty$ as $r \rightarrow \infty$. Throughout this paper the intervals determined by $\theta$ will be denoted by $I_{r}:=\left(k_{r-1}-k_{r}\right]$, and the ratio $k_{r} / k_{r-1}$ will be abbreviated by $q_{r}$ (see Fredman et al[8]). Recently, Aydin[1] defined the concept of almost lacunary convergent as follow: A bounded sequence $x=\left(x_{k}\right)$ is said be almost lacunary convergent to the number $\ell$ if and only if

$\lim _{r} \frac{1}{h_{r}} \sum_{j \in I_{r}} x_{j+n}=\ell$, uniformly in $\mathrm{n}$.

The idea of $\sigma$-convergence for double sequences was introduced in [4] and further studied recently in [3] and [15]. In [11]-[14] we study various classes of four dimensional matrices, e.g. $\sigma$-regular, $\sigma$-conservative, regularly $\sigma$-conservative, boundedly $\sigma$-conservative and $\sigma$-coercive matrices.

In this paper, we define $(\sigma, \theta)$-convergence. We also generalize the above matrices by characterizing the $(\sigma, \theta)$-conservative, $(\sigma, \theta)$-regular and $(\sigma, \theta)$ coercive matrices. Further, we also determine the associated bounded linear operators for these matrix classes. which is the generalized result of Mursaleen, M.A. Jarrah and S.Mouhiddin see ref [15]

\section{2. $(\sigma, \theta)$-Lacunary convergent sequences}

We define the following:

Definition 2.1. [ sir paper,2009 ]A bounded sequence $x=\left(x_{k}\right)$ of real numbers is said to be $(\sigma, \theta)$-lacunary convergent to a number $\ell$ if and only if $\lim _{r} \frac{1}{h_{r}} \sum_{j \in I_{r}} x_{\sigma^{j}(n)}=\ell$, uniformly in $\mathrm{n}$, and let $V_{\sigma}(\theta)$, denote the set of all such sequences, i.e where

$$
V_{\sigma}(\theta)=\left\{x \in \ell_{\infty}: \lim _{r} \frac{1}{h_{r}} \sum_{j \in I_{r}} x_{\sigma^{j}(n)}=\ell, \text { uniformly in } n\right\}
$$

Note that for $\sigma(n)=n+1, \sigma$ - lacunary convergence is reduced to almost lacunary convergence. Results similar to that Aydin[1] can easily be proved for the space $V_{\sigma}(\theta)$,

Definition 2.2. A bounded sequence $x=\left(x_{k}\right)$ of real numbers is said to be $\sigma$-lacunary bounded if and only if $\sup _{r, n}\left|\frac{1}{h_{r}} \sum_{j \in I_{r}} x_{\sigma^{j}(n)}\right|<\infty$, and we let $V_{\sigma}^{\infty}(\theta)$, denot the set of all such sequences

$$
V_{\sigma}^{\infty}(\theta)=\left\{x \in \ell_{\infty}: \sup _{r, n}\left|\tau_{r, n}(x)\right|<\infty\right\} .
$$


Where

$$
\tau_{r n}(x)=\frac{1}{h_{r}} \sum_{j \in I_{r}} x_{\sigma^{j}(n)},
$$

Note that $c \subset V_{\sigma}(\theta) \subset V_{\sigma}^{\infty}(\theta) \subset \ell_{\infty}$.

Definition 2.3. An infinite matrix $A=\left(a_{n k}\right)$ is said to be $(\sigma, \theta)$-conservative if and only if $A x \in V_{\sigma}(\theta)$ for all $x=\left(x_{k}\right) \in c$ and we denote this by $A \in\left(c, V_{\sigma}(\theta)\right)$.

Definition 2.4. We say that, infinite matrix $A=\left(a_{n k}\right)$ is said to be $(\sigma, \theta)$-regular if and only if it is $V_{\sigma}(\theta)$-conservative and $(\sigma, \theta)-\lim A x=$ $\lim x$ for all $x \in c$ and we denote this by $A \in\left(c, V_{\sigma}(\theta)\right)_{\text {reg }}$.

Definition 2.5. A matrix $A=\left(a_{n k}\right)$ is said to be $(\sigma, \theta)$-coercive if and only if $A x \in V_{\sigma}(\theta)$ for all $x=\left(x_{k}\right) \in \ell_{\infty}$ and we denote this by $A \in\left(\ell_{\infty}, V_{\sigma}(\theta)\right)$. Remark 2.6. If we take $h_{r}=r$ then $V_{\sigma}(\theta)$ is reduced to the space $V_{\sigma}$ and $(\sigma, \theta)$-conservative, $(\sigma, \theta)$-regular, $(\sigma, \theta)$-coercive matrices are respectively reduced to $\sigma$-conservative, $\sigma$-regular, $\sigma$-coercive matrices (cf [15]); and in addition if $\sigma(n)=n+1$ then the space $V_{\sigma}(\theta)$ is reduced to the space $f$ of almost convergent sequences ( $\mathrm{cf}[9]$ ) and these matrices are reduced to the almost conservative, almost regular ( $\mathrm{cf}[7]$ ) and almost coercive matrices respectively (cf $[6])$.

\section{3. $(\sigma, \theta)$-conservative matrices and bounded linear operators}

In the following theorem we characterize $(\sigma, \theta)$-conservative matrices and find the associated bounded linear operator.

Theorem 3.1. A matrix $A=\left(a_{n k}\right)$ is $(\sigma, \theta)$-conservative, i.e. $A \in$ $\left(c, V_{\sigma}(\theta)\right)$ if and only if it satisfies the condition

(i) $\|A\|=\sup _{n} \sum_{k}\left|a_{n k}\right|<\infty$;

(ii) $a_{(k)}=\left(a_{n k}\right)_{n=1}^{\infty} \in V_{\sigma}(\theta)$, for each $k$;

(iii) $a=\left(\sum_{k} a_{n k}\right)_{n=1}^{\infty} \in V_{\sigma}(\theta)$.

In this case, the $(\sigma, \theta)$-limit of $A x$ is $\lim x\left[u-\sum_{k} u_{k}\right]+\sum_{k} x_{k} u_{k}$, where $u=(\sigma, \theta)-\lim a$ and $u_{k}=(\sigma, \theta)-\lim a_{k}, k=1,2, \cdots$ 
Proof. Sufficiency. Let the conditions hold. Let $r$ be any non-negative integer and $x=\left(x_{k}\right) \in c$. For every positive integer $n$; write $\tau_{r n}(x)=$ $\frac{1}{h_{r}} \sum_{k=1}^{\infty} \sum_{j \in I_{r}} a_{\sigma^{j}(n), k} x_{k}$ Then we have $-\tau_{r n}(x)\left|\leq \frac{1}{h_{r}} \sum_{k=1}^{\infty} \sum_{j \in I_{r}}\right| a_{\sigma^{j}(n), k}|| x_{k} \mid$ $\leq \frac{\|x\|}{h_{r}} \sum_{k=1}^{\infty} \sum_{j \in I_{r}}\left|a_{\sigma^{j}(n), k}\right| \leq\|A\|\|x\|$. Since $\tau_{r n}$ is obviously linear on $c$, it follows that $\tau_{r n} \in c^{\prime}$ and $\left\|\tau_{r n}\right\| \leq\|A\|$.

Now, $\tau_{r n}(e)=\frac{1}{h_{r}} \sum_{k=1}^{\infty} \sum_{j \in I_{r}} a_{\sigma^{j}(n), k}=\frac{1}{h_{r}} \sum_{j \in I_{r}} \sum_{k=1}^{\infty} a_{\sigma^{j}(n), k}$ that is, $\lim _{r} \tau_{r n}(e)$ exists uniformly in $n$ and $\lim _{r} \tau_{r n}(e)=u$ uniformly in $n$, the $(\sigma, \theta)$-limit of $a$, since $a \in V_{\sigma}(\theta)$. Similarly, $\lim _{r} \tau_{r n} e^{k}=u_{k}$, the $(\sigma, \theta)$ limit of $a_{(k)}$ for each $k$, uniformly in $n$. Since $\left\{e, e^{1}, e^{2}, \cdots\right\}$ is a fundamental set in $c$, and $\sup _{r}\left|\tau_{r, n}(x)\right|$ is finite for each $x \in c$, it follows that $\lim _{r} \tau_{r n}(x)=\tau_{n}(x)$, exists for all $x \in c(\operatorname{cf}[5])$. Furthermore, $\left\|\tau_{n}\right\| \leq$ $\liminf _{r} \stackrel{r}{\|} \tau_{r n}\|\leq\| A \|$ for each $n$ and $\tau_{n} \in c^{\prime}$. Thus, each $x \in c$ has a unique representation $\mathrm{x}=(\lim x)\left[e-\sum_{k} e_{k}\right]+\sum_{k} x_{k} e_{k} \tau_{n}(x)=(\lim x)\left[t_{n}(e)-\right.$ $\left.\sum_{k} t_{n}\left(e_{k}\right)\right]+\sum_{k} x_{k} t_{n}\left(e_{k}\right) \tau_{n}(x)=(\lim x)\left[u-\sum_{k} u_{k}\right]+\sum_{k} x_{k} u_{k}$. By $L(x)$, we denote the right hand side of the above expression which is independent of $n$. Now, we have to show that $\lim _{r} \tau_{r n}(x)=L(x)$ uniformly in $n$. Put $\mathrm{F}_{r n}(x)=\tau_{r n}(x)-L(x)$. Then $F_{r n} \in c^{\prime},\left\|F_{r n}\right\| \leq 2\|A\|$ for all $r, n$, $\lim _{r} F_{r n}(e)=0$ uniformly in $n$, and $\lim _{r} F_{r n}\left(e^{k}\right)=0$ uniformly in $n$ for each $k$. Let $K$ be an arbitrary positive integer. Then $\mathrm{x}=(\lim x) e+\sum_{k=1}^{K}\left(x_{k}-\right.$ $\lim x) e^{k}+\sum_{k=K+1}^{\infty}\left(x_{k}-\lim x\right) e^{k}$.Now applying $F_{r n}$ on both sides of the above equality, we have $\mathrm{F}_{r n}(x)=(\lim x) F_{r n}(e)+\sum_{k=1}^{K}\left(x_{k}-\lim x\right) F_{r n}\left(e^{k}\right)+$ $F_{r n}\left(\sum_{k=K+1}^{\infty}\left(x_{k}-\lim x\right) e^{k}\right) \cdot(3.1 .1)$ Now, $\left|F_{r n}\left(\sum_{k=K+1}^{\infty}\left(x_{k}-\lim x\right) e^{k}\right)\right| \leq$ $2\|A\| \sum_{k \geq K+1}\left\{\left|x_{k}-\lim x\right|\right\}$, for all $r, n$. After choosing fixed $K$ large enough, it is easy to see that the absolute value of each term on the right hand side of (3.1.1) can be made uniformly small for all sufficiently large $r$. Therefore, $\lim _{r} F_{r n}(x)=0$ uniformly in $n$; so that $A x \in V_{\sigma}(\theta)$ and the matrix $A$ is $(\sigma, \theta)$-conservative.

Necessity. Suppose that $A$ is $(\sigma, \theta)$-conservative. Then $\mathrm{Ax}=\left(\mathrm{A}_{n}(x)\right)_{n=1}^{\infty}=$ $\left(\sum_{k} a_{n k} x_{k}\right)_{n=1}^{\infty} \in V_{\sigma}(\theta)$, for all $x \in c$. Let $x=\left(x_{k}\right)=e^{k}$. Therefore $(\sigma, \theta)-\lim _{n} \sum_{k}^{n=1} a_{n k} e^{k}=(\sigma, \theta)-\lim _{n} a_{n k}=a_{(k)}$. Hence (ii) holds. Now, let $x=e$. Then $(\sigma, \theta)-\lim _{n} \sum_{k} a_{n k} e=(\sigma, \theta)-\lim _{n} \sum_{k} a_{n k}=a$, so that (iii) must hold. Since $A x=\left(A_{n}(x)\right) \in V_{\sigma}(\theta) \subset \ell_{\infty}$. It follows that $\sup _{n}\left|A_{n}(x)\right|<$ 
$\infty,\left(A_{n}\right)$ is a sequence of bounded operators. Therefore, by Banach-Steinhaus

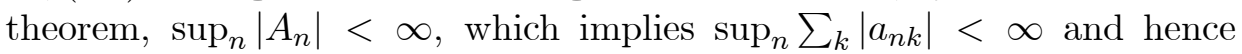
$\|A\|=\sup _{n} \sum_{k}\left|a_{n k}\right|<\infty$, i.e. (i).

This completes the proof of the theorem.

Now, we deduce the following.

Corollary 3.2. $A=\left(a_{n k}\right)$ is $(\sigma, \theta)$-regular if and only if the conditions (i), (ii) with $(\sigma, \theta)$-limit zero for each $k$, and (iii) with $(\sigma, \theta)$-limit 1 of Theorem 3.1 hold.

Proof. For $x \in c,(\sigma, \theta)-\lim A x=L(x)$, which reduces to $\lim x$, since $u=1$ and $u_{k}=0$ for each $k$. Hence $A$ is $(\sigma, \theta)$-regular.

Conversely, let $A$ be $(\sigma, \theta)$-regular. Then $(\sigma, \theta)-\lim A e=1=(\sigma, \theta)$ $\lim A a,(\sigma, \theta)-\lim A e^{k}=0=(\sigma, \theta)-\lim A_{(k)}$ and $\|A\|$ is finite as condition (i) of Theorem 3.1.

This completes the proof of the Corollary 3.2.

4. $(\sigma, \theta)$-coercive matrices

We use the following lemma in our next theorem.

Lemma 4.1. Let $B(n)=\left(b_{m k}(n)\right), n=0,1,2, \cdots$ be a sequence of infinite matrices such that

(i) $\|B(n)\|<H<+\infty$ for all $n$; and

(ii) $\lim _{m} b_{m k}(n)=0$ for each $k$, uniformly in $n$.

Then $\lim _{m} \sum_{k} b_{m k}(n) x_{k}=0$ uniformly in $n$ for each $x \in \ell_{\infty}(4.1 .1)$ if and only if $\lim _{m} \sum_{k}\left|b_{m k}(n)\right|=0$ uniformly in $n .(4.1 .2)$

Theorem 4.2. A matrix $A=\left(a_{n k}\right)$ is $(\sigma, \theta)$-coercive, i.e. $A \in\left(\ell_{\infty}, V_{\sigma}(\theta)\right)$ if and only if (i) and (ii) of Theorem 3.1 hold, and

(iii) $\lim _{r} \sum_{k=1}^{\infty}\left|\sum_{j \in I_{r}} a_{\sigma^{j}(n), k}-u_{k}\right|$ uniformly in $n$.

In this case, the $(\sigma, \theta)$-limit of $A x$ is $\sum_{k} x_{k} u_{k} \quad \forall x \in \ell_{\infty}$, where $u_{k}=$ $(\sigma, \theta)-\lim a_{k}$.

Proof. Sufficiency. Let the conditions hold. For any positive integer $K \quad \sum_{k=1}^{K}\left|u_{k}\right|=\sum_{k=1}^{K} \lim _{r}\left|\sum_{j \in I_{r}} a_{\sigma^{j}(n), k}\right| / h_{r}=\lim _{r} \sum_{k=1}^{K}\left|\sum_{j \in I_{r}} a_{\sigma^{j}(n), k}\right| / h_{r} \leq$ 
$\lim \sup _{r} \sum_{j \in I_{r}} \sum_{k=1}^{\infty}\left|a_{\sigma^{j}(n), k}\right| / h_{r} \leq\|A\|$. This shows that $\sum_{k=1}^{\infty}\left|u_{k}\right|$ converges, and that $\sum_{k=1}^{\infty} u_{k} x_{k}$ is defined for every $x=\left(x_{k}\right) \in \ell_{\infty}$.

Let $x=\left(x_{k}\right)$ be any arbitrary bounded sequence. For every positive integer $r\left\|\sum_{k=1}^{\infty}\left(\frac{1}{h_{r}} \sum_{j \in I_{r}} a_{\sigma^{j}(n), k}-u_{k}\right) x_{k}\right\|=\left\|\sum_{k=1}^{\infty}\left[\sum_{j \in I_{r}}\left[a_{\sigma^{j}(n), k}-u_{k}\right] / h_{r}\right] x_{k}\right\|$ $\leq \sup _{n}\left[\left|\sum_{k=1}^{\infty}\left[\sum_{j \in I_{r}}\left[a_{\sigma^{j}(n), k}-u_{k}\right] / h_{r}\right] x_{k}\right|\right] \leq\|x\| \sup _{r}\left[\sum_{k=1}^{\infty} \mid \sum_{j \in I_{r}}\left[a_{\sigma^{j}(n), k}-\right.\right.$ $\left.\left.u_{k}\right] \mid / h_{r}\right]$.

Letting $r \rightarrow \infty$ and using condition (iii), we get

$$
\frac{1}{h_{r}} \sum_{k=1}^{\infty} \sum_{j \in I_{r}} a_{\sigma^{j}(n), k} x_{k} \longrightarrow \sum_{k=1}^{\infty} u_{k} x_{k} .
$$

Hence $A x \in V_{\sigma}(\theta)$ with $(\sigma, \theta)$-limit $\sum_{k=1}^{\infty} u_{k} x_{k}$.

Necessity. Let $A$ be $(\sigma, \theta)$-coercive matrix. This implies that $A$ is $(\sigma, \theta)$ conservative, then we have condition (i) and (ii) from Theorem 3.1. Now we have to show that (iii) holds.

Suppose that for some $n$, we have $\lim \sup _{r} \sum_{k=1}^{\infty}\left|\sum_{j \in I_{r}}\left[a_{\sigma^{j}(n), k}-u_{k}\right]\right| / h_{r}=$ $N>0$.Since $\|A\|$ is finite, therefore $N$ is also finite. We observe that since $\sum_{k=1}^{\infty}\left|u_{k}\right|<+\infty$ and $A$ is $(\sigma, \theta)$-coercive, the matrix $B=\left(b_{n k}\right)$, where $b_{n k}=a_{n k}-u_{k}$, is also $(\sigma, \theta)$-coercive matrix. By an argument similar to that of Theorem 2.1 in [6], one can find $x \in \ell_{\infty}$ for which $B x \notin V_{\sigma}(\theta)$. This contradiction implies the necessity of (iii).

Now, we use Lemma 4.1 to show that this convergence is uniform in $n$. Let $\mathrm{t}_{r k}(n)=\sum_{j \in I_{r}}\left[a_{\sigma^{j}(n), k}-u_{k}\right] / h_{r}$ and let $T(n)$ be the matrix $\left(t_{r k}(n)\right)$. It is easy to see that $\|H(n)\| \leq 2\|A\|$ for every $n$; and from condition (ii) $\lim _{r} t_{r k}(n)=0$ for each $k$, uniformly in $n$.For any $x \in \ell_{\infty}$ $\lim _{r} \sum_{j \in I_{r}} t_{r k}(n) x_{k}=(\sigma, \theta)-\lim A x-\sum_{k=1}^{\infty} u_{k} x_{k}$ and the limit exists uniformly in $n$, since $A x \in V_{\sigma}(\theta)$. Moreover, this limit is zero since $\left|\sum_{k=1}^{\infty} t_{r k}(n) x_{k}\right| \leq$ 
$\|x\| \sum_{k=1}^{\infty}\left|\sum_{j \in I_{r}}\left[a_{\sigma^{j}}(n), k-u_{k}\right]\right| / h_{r}$. Hence $\lim _{r} \sum_{k=1}^{\infty}\left|t_{r k}(n)\right|=0$ uniformly in $n$; i.e. the condition (iii) holds.

This completes the proof of the theorem.

Acknowledgement: I would like to thank to the Deanship of scientific research for supporting the research project 14/2011.

\section{References}

[1] B. Aydin,Lacunary almost summability in certain linear topological spaces, Bull. Malays Math.Sci.Soc. 2, pp. 217-223, (2004).

[2] S.Banach, Theorie des operations lineaires (Warsaw), (1932).

[3] C. Çakan, B. Altay and H. Çoşkun, $\sigma$-regular matrices and a $\sigma$-core theorem for double sequences, Hacettepe J. Math. Stat., 38 (1), pp. 51-58, (2009).

[4] C. Cakan, B. Altay and M. Mursaleen, The $\sigma$-convergence and $\sigma$-core of double sequences, Applied Mathematics Letters, 19, pp. 1122-1128, (2006).

[5] N. Dunford and J. T. Schwartz, Linear Operators: General theory, Pure and Appl. Math., Vol. 7, Interscience, New York, (1958).

[6] C. Eizen and G. Laush, Infinite matrices and almost convergence, Math. Japon., 14, pp. 137-143, (1969).

[7] J. P. King, Almost summable sequences, Proc. Amer. Math. Soc., 17, pp. 1219-1225, (1966).

[8] A. R. Freedman,J.J. Sember and M. Raphael, Some cesaro type summability space, Proc London Math.Soc. 37, pp. 508-520, (1978).

[9] G. G. Lorentz, A contribution to theory of divergent sequences, Acta Math., 80, pp. 167-190, (1948).

[10] M. Mursaleen, On some new invariant matrix methods of summability, Quart. J. Math. Oxford, 34 (1983), 77-86. 
[11] M. Mursaleen, Some matrix transformations on equence space of invariant means, Hacettpe. J. Math and Stat, 38(3) (2009), 259-264, (2009).

[12] M. Mursaleen and S.A.Mohiuddine, Double $\sigma$-multiplicative matrices, J. Math. Anal. Appl., 327, pp. 991-996, (2007).

[13] M. Mursaleen and S.A.Mohiuddine, Regularly $\sigma$-conservative and $\sigma$ coercive four dimensional matrices, Computers and Mathematics with Applications, 56, pp. 1580-1586, (2008).

[14] M. Mursaleen and S.A.Mohiuddine, On $\sigma$-conservative and boundedly $\sigma$-conservative four dimensional matrices, Computers and Mathematics with Applications, 59, pp. 880-885, (2009).

[15] M. Mursaleen and S.A.Mohiuddine, Some inequalities on sublinear functionals related to the invariant mean for double sequences, Math. Ineq. Appl.,139(1), pp. 157-163, (2010).

[16] M. Mursaleen and S.A.Mohiuddine, Some new double sequence spaces of invariant means, Glasnik Matematicki, 45(1), pp. 139-153, (2010).

[17] P. Schaefer, Infinite matrices and invariant means, Proc. Amer. Math. Soc., 36, pp. 104-110, (1972).

\author{
M. Aiyub \\ Department of Mathematics, \\ University of Bahrain, \\ P.O. Box-32038, \\ Kingdom of Bahrain \\ e-mail : maiyub2002@yahoo.com
}

